\title{
Sputum cell count: biomarkers in the differentiation of asthma, COPD and asthma-COPD overlap
}

\author{
This article was published in the following Dove Press journal: \\ International Journal of COPD \\ II September 2017 \\ Number of times this article has been viewed
}

\section{Jie Gao \\ Wutie Zhou \\ Bida Chen \\ Weiming Lin \\ Sifang Wu \\ Feng Wu}

Department of Respiratory Medicine, The Third People's Hospital, Guangzhou Medical College, Huizhou, People's Republic of China
Correspondence: Feng Wu

Department of Respiratory Medicine,

The Third People's Hospital, Guangzhou

Medical College, I\# Xuebei Avenue,

Huizhou 516002, Guangdong, People's

Republic of China

$\mathrm{Tel}+86$ I35 $02436 \mid 32$

Fax +86752 2359825

Email huizhouwufeng@।26.com
Introduction: Cell count in induced sputum is a noninvasive biomarker to assess airway inflammation phenotypes. Accordingly, sputum cell counts are extensively used in the treatment of asthma and COPD. Nevertheless, the clinical application of sputum cell counts in patients with asthma-COPD overlap (ACO) remains elusive. The aim of this study was to investigate sputum cell counts in patients with ACO which are different from those in patients with asthma and COPD and also to examine the relationship between sputum cell counts in bronchial reversibility and bronchial hyperresponsiveness (BHR).

Patients and methods: A total of 374 patients participated in the study, including 142 patients with asthma, 160 patients with COPD and 72 patients with ACO. All patients underwent the following tests on the same day: pulmonary function test (PFT), BHR test or bronchodilator reversibility test and inducing sputum. They were classified into the asthma group, COPD group or ACO group based on a clinical history, PFT values and BHR test or bronchodilator reversibility test.

Results: The three groups had different PFT values $(p<0.001)$ except for forced vital capacity (FVC) between the asthma and ACO groups ( $p=0.378$ ). The sputum levels of eosinophil\% were decreased in patients with COPD when compared with those in patients with asthma and $\operatorname{ACO}(p<0.001$ and $p<0.001$, respectively). There was a difference in sputum neutrophil $\%$ and macrophage $\%$ counts among the three groups ( $p<0.001$ and $p<0.001$, respectively); there was no difference in sputum eosinophil $\%$ counts between patients with ACO and asthma $(p=0.668)$ and there was no difference in the percentage of induced sputum cells between the stage of airway obstruction and the stage of BHR.

Conclusion: The clinical relevance of this study provides evidence that sputum cell counts as an inflammatory biomarker could carry some information to distinguish ACO, asthma and $\mathrm{COPD}$, and these biomarkers need more studies to provide diagnostic value in the differentiation between ACO, asthma and COPD.

Keywords: induced sputum cell counts, COPD, BHR, bronchodilator reversibility, asthma, asthma-COPD overlap

\section{Introduction}

Asthma and COPD are considered as chronic airway inflammatory diseases, which are typically associated with Th2-mediated immune response in asthma and related to the characteristics of Th1-mediated immunity in COPD. ${ }^{1}$ Asthma-COPD overlap (ACO) is the term to describe patients who have features of both asthma and COPD, recommended by the 2017 Global Initiative for Asthma (GINA) and the Global Initiative for Chronic Obstruction Lung Disease (GOLD) guidelines. Patients with asthma and COPD are commonly seen in clinical practice. In addition, compared with patients with asthma or COPD alone, patients with ACO experience frequent exacerbations and hospitalizations, have poor quality of life, a more rapid decline in lung function 
and high mortality, and consume a disproportionate amount of health care resources than asthma or COPD alone. ${ }^{2,3}$

As asthma and COPD are heterogeneous diseases, each with a range of underlying mechanisms, ACO does not represent a single disease or phenotype. The clinical phenotypes and underlying mechanisms of ACO remain controversial, and there is no consensus on the best definition of ACO. Assessment of airway inflammation is normally recognized as an important aspect in the diagnosis and clinical control between asthma and COPD. Measurement of airway eosinophilia is a reliable predictive index of responsiveness to inhaled and oral corticosteroid therapies in patients with asthma and COPD. In addition, airway inflammation can be measured through the airway noninvasively by induced sputum analysis. In 2016, the normal reference values of induced sputum cytology in the People's Republic of China were defined as sputum eosinophils $\geq 2.5 \%$, which was identified as airway eosinophilia. ${ }^{4}$ The biomarkers are considered as a direct, reliable, sensitive, simple and repeatable method of assessing airway inflammatory phenotypes, widely used in clinical practice. However, the significance of these inflammatory biomarkers in the diagnosis and therapy of ACO remains unclear.

We conducted a cross-sectional study to 1) evaluate the diagnostic performance of induced sputum analysis, which may be able to indicate airway eosinophilic/neutrophilic inflammation in patients in the differentiation of asthma, COPD and ACO and 2) assess the relationship between sputum eosinophils, bronchial hyperresponsiveness (BHR) and bronchodilator reversibility in asthma and ACO.

\section{Patients and methods Significance of study}

The aim of the study was to evaluate the diagnostic performance of induced sputum analysis in the differentiation of asthma, COPD and ACO, and assess the relationship between sputum eosinophils, BHR and bronchial reversibility in asthma and ACO. Data were collected during regular clinical practice and medical procedures and analyzed retrospectively.

\section{Subjects}

Patients diagnosed with asthma, COPD or ACO and had visited the Third People's Hospital of Guangzhou Medical College in Huizhou from May 2016 to April 2017 were retrospectively enrolled in the study. The GOLD stage of COPD was defined according to the 2017 recommendations of GOLD, and the BHR stage of asthma and cumulative dose causing a $20 \%$ decrease in forced expiratory volume in 1 second $\left(\mathrm{FEV}_{1} ; \mathrm{PD}_{20}\right.$ [provocative dose]) at baseline was determined by the 2014 recommendations of the Chinese National Guidelines of Pulmonary Function Test. ${ }^{5}$

Patients were divided into three study groups: asthma, COPD or ACO.

Asthma patients were diagnosed according to a clinical history of wheezing, cough, chest tightness or shortness of breath, as well as the presence of BHR (cumulative dose causing a $20 \%$ decrease in $\mathrm{FEV}_{1}$ ), based on the GINA guidelines. None of them had a history of COPD, or previous doctordiagnosed ACO. All subjects had not used any oral or/and inhaled corticosteroid (ICS) in the previous 12 weeks. The included patients with asthma had initial diagnosis and were under uncontrolled stage.

COPD patients were diagnosed according to the GOLD criterion, which included a post-bronchodilator spirometry to confirm airflow obstruction $\left(\mathrm{FEV}_{1}\right.$ to forced vital capacity ratio $\left[\mathrm{FEV}_{1} / \mathrm{FVC}\right]<70 \%$ ), in a clinical context (dyspnea, chronic cough or sputum production, and a history of exposure to risk factors for the disease). They had received a COPD diagnosis at least 1 year before the study. None of them had a history of asthma. All subjects had not used any oral or/and ICS in the previous 4 weeks. The included COPD patients had exacerbations.

There are currently no internationally accepted criteria for the diagnosis of ACO. Thus, in this study, we defined ACO patients according to the 2017 GINA/GOLD document 1) the presence of clinical characteristics of asthma and COPD 2) a post-bronchodilator spirometry reading confirming airflow obstruction $\left(\mathrm{FEV}_{1} / \mathrm{FVC}<70 \%\right)$; and 3$)$ a post-bronchodilator increase in $\mathrm{FEV}_{1}>12 \%$ and $200 \mathrm{~mL}$ from baseline, or increase in $\mathrm{FEV}_{1}>12 \%$ and $200 \mathrm{~mL}$ from baseline after a week of anti-inflammatory treatment. The degree of ACO patients' airway obstruction was defined by the GOLD stage of COPD. All subjects had not used any oral or/ and ICS in the previous 12 weeks. The included patients with ACO had exacerbations and were under uncontrolled stage.

We excluded patients if they had confounding pulmonary comorbidities, such as pulmonary tuberculosis, interstitial lung disease, lung cancer or pulmonary infection, and had a cognitive impairment that may affect the collaboration or comprehension of the study.

\section{Ethics statement}

The institutional review board of the Third People's Hospital of Guangzhou Medical College in Huizhou approved the study protocol and absolved the need for written informed 
consent from patients as the study was a retrospective study with personal identification data anonymized.

\section{Assessments and study procedures}

On the same day, the following tests or determinations were performed: pulmonary function test (PFT), BHR test or bronchodilator reversibility test and induced sputum. Clinical variables were recorded in participants.

\section{PFT}

Airway limitation was identified using lung function machine (MS-pneumo + aps; Jaeger, Friedberg, German) by an experienced technician according to the 2014 recommendations of the Chinese National Guidelines of Pulmonary Function Test. Percentage predicted values (\%pred) were calculated based on reference values for healthy Chinese adults. All patients were required to undergo PFT in a reproducible way, and the best values were retained. ${ }^{5}$

\section{BHR test}

PFT values were assessed prior to the methacholine challenge. Patients with a $\mathrm{FEV}_{1} \%$ pred $<60 \%$ were excluded from the BHR test (at baseline). The breath dosimeter method was used according to published guidelines from Chinese National Guidelines of Pulmonary Function Test. ${ }^{5}$ The test sequence included five steps: $0.9 \% \mathrm{NaCl}$ only, $0.078,0.312$, 1.125 and $2.504 \mathrm{mg}$. Airway responsiveness was expressed as the $\mathrm{PD}_{20}$ of methacholine (causing a $20 \%$ reduction in $\mathrm{FEV}_{1}$ ) required to induce a $20 \%$ decrease in $\mathrm{FEV}_{1}\left(\mathrm{PD}_{20}\right)$, and the positive response was defined as $\mathrm{PD}_{20} \leq 2.504 \mathrm{mg}$ (between NS and $2.504 \mathrm{mg}) .^{5}$

\section{Bronchodilator reversibility test}

Patients were asked to inhale $400 \mu \mathrm{g}$ salbutamol via a metereddose inhaler after baseline evaluation, and PFT was repeated not less than 20 minutes. Three forced expiratory maneuvers were recorded. Postbronchodilator $\mathrm{FEV}_{1} / \mathrm{FVC}<70 \%$ and the positive response, which was defined as $\mathrm{FEV}_{1}>12 \%$ and $200 \mathrm{~mL}$ after salbutamol inhalation, were obtained. ${ }^{5}$

\section{Sputum induction}

Sputum was induced with hypertonic saline inhalation through ultrasonic atomizer. A single hypertonic saline $(3 \% \mathrm{NaCl})$ was used. Patients were asked to inhale $400 \mu \mathrm{g}$ salbutamol via a metered-dose inhaler 20 minutes before induction. Collected lower respiratory sputum portions of induced sputum were dispersed using $0.1 \%$ dithiothreitol in a water bath $\left(37^{\circ} \mathrm{C}\right)$ and oscillator 15 minutes before the 300 mesh nylon mesh filter.
Subsequently, total cell count was centrifuged, smeared and stained (hematoxylin-eosin). A differential cell count was obtained from 400 cells under $400 \times$ microscope to identify the severity and type of airway inflammation in patients with COPD. Oral squamous cell count less than $10 \%$ in each sample was deemed adequate for further analysis. We defined sputum eosinophilic percentage of $\geq 2.5 \%$ as abnormal. ${ }^{4}$

\section{Statistical analysis}

Analysis of the data was performed using SPSS 19 (IBM Corporation, Armonk, NY, USA). Continuous variables are expressed as mean $\pm \mathrm{SD}$ or median (interquartile range) for non-normal variables when appropriate, and categorical variables as frequencies and percentages with the chi-square test. Comparisons between groups were analyzed using the ANOVA test for quantitative variables and the Kruskal-Wallis test for categorical variables. The relationship between sputum eosinophils, BHR and bronchodilator reversibility was assessed using the Spearman's rank correlation coefficient. Correlation between tests was performed by constructing receiver operating characteristic (ROC) curve. The optimal cutoff value was determined from the highest sum of sensitivity and specificity. Statistical significance was defined as $p<0.05$.

\section{Results}

\section{Characteristics of the patients}

Patient demographic information is presented in Table 1. A total of 374 patients were included in this study. According

Table I Patient demographics and baseline characteristics

\begin{tabular}{|c|c|c|c|c|}
\hline $\begin{array}{l}\text { Demographic } \\
\text { parameter }\end{array}$ & $\begin{array}{l}\text { Asthma } \\
(\mathrm{N}=142)\end{array}$ & $\begin{array}{l}\text { COPD } \\
(N=160)\end{array}$ & $\begin{array}{l}\text { ACO } \\
(\mathrm{N}=72)\end{array}$ & $p$-value \\
\hline Mean age, years (SD) & $46.2(16.45)$ & $71.8(8.50)$ & 59.5 (15.78) & $<0.001$ \\
\hline Male, n (\%) & $56(39.4)$ & $132(82.5)$ & $53(73.6)$ & $<0.00 I^{*}$ \\
\hline Race, Chinese, n (\%) & 100 & 100 & 100 & \\
\hline Mean height, cm (SD) & |6I (8.42) & $160(7.46)$ & $163(7.83)$ & 0.840 \\
\hline Mean weight, kg (SD) & $59.8(10.58)$ & $52.5(10.20)$ & $59.8(10.36)$ & 0.999 \\
\hline Mean body mass & $23.19(3.78)$ & $20.5 I(3.5 I)$ & $22.36(3.16)$ & 0.270 \\
\hline \multicolumn{5}{|l|}{ index, $\mathrm{kg} / \mathrm{m}^{2}(\mathrm{SD})$} \\
\hline Smokers, n (\%) & $24(16.9)$ & $112(70)$ & $37(5 \mathrm{I} .4)$ & $<0.007$ \\
\hline \multicolumn{5}{|l|}{ GOLD stage, n (\%) } \\
\hline I & - & $2(1.25)$ & $8(I I . I I)$ & \\
\hline 2 & - & $49(30.62)$ & $50(69.45)$ & \\
\hline 3 & - & $58(36.25)$ & 14 (19.44) & \\
\hline 4 & - & $51(31.88)$ & $0(0)$ & \\
\hline \multicolumn{5}{|l|}{ BHR stage, n (\%) } \\
\hline Very mild & $36(25.35)$ & - & - & \\
\hline Mild & $37(26.06)$ & - & - & \\
\hline Moderate & $48(33.8)$ & - & - & \\
\hline Severe & $21(14.79)$ & - & - & \\
\hline
\end{tabular}

Notes: $\mathrm{N}$ refers to the total population; $\mathrm{n}$ refers to the subgroup population. *Difference between two groups was not statistically significant $(p>0.05)$.

Abbreviations: ACO, asthma-COPD overlap; BHR, bronchial hyperresponsiveness; GOLD, Global Initiative for Chronic Obstruction Lung Disease. 
Table 2 Spirometry results of the patients

\begin{tabular}{|c|c|c|c|c|}
\hline Variable & $\begin{array}{l}\text { Asthma (N=142) } \\
\text { (baseline) }\end{array}$ & $\begin{array}{l}\text { COPD }(\mathrm{N}=160) \\
\text { (postbronchodilator) }\end{array}$ & $\begin{array}{l}\text { ACO }(\mathrm{N}=72) \\
\text { (postbronchodilator) }\end{array}$ & $p$-value \\
\hline $\mathrm{FVC}(\mathrm{L})$, mean $(\mathrm{SD})$ & $3.21(0.82)$ & $1.97(0.66)$ & $3.04(0.82)$ & $<0.00 I^{*}$ \\
\hline $\mathrm{FEV}_{1}(\mathrm{~L})$, mean $(\mathrm{SD})$ & $2.34(0.68)$ & $0.93(0.4 \mathrm{I})$ & $1.66(0.58)$ & $<0.001$ \\
\hline FEV , \%predicted, mean (SD) & $85.55(12.83)$ & $42.39(18.90)$ & $61.97(15.33)$ & $<0.001$ \\
\hline $\mathrm{FEV}_{\mathrm{I}} / \mathrm{FVC}(\%)$, mean (SD) & $73(9.58)$ & 46.85 (I I.44) & $54.04(9.61)$ & $<0.001$ \\
\hline PEF (L/min), mean (SD) & $6.04(1.85)$ & $2.53(1.25)$ & $4.15(1.62)$ & $<0.001$ \\
\hline MMEF (L/sec), mean (SD) & $\mathrm{I} .87(0.45)$ & $0.47(0.24)$ & $0.87(0.45)$ & $<0.001$ \\
\hline $\mathrm{MEF}_{50 \%}(\mathrm{~L} / \mathrm{sec})$, mean (SD) & $2.22(1.01)$ & $0.54(0.31)$ & $1.07(0.54)$ & $<0.001$ \\
\hline $\operatorname{MEF}_{25 \%}(\mathrm{~L} / \mathrm{sec})$, mean $(\mathrm{SD})$ & $1.02(1.08)$ & $0.24(0.12)$ & $0.4(0.2 \mathrm{I})$ & $<0.001$ \\
\hline
\end{tabular}

Note: *Difference between two groups was not statistically significant $(p>0.05)$.

Abbreviations: ACO, asthma-COPD overlap; $\mathrm{FEV}_{1}$, forced expiratory volume in I second; FVC, forced vital capacity; MEF, maximal expiratory flow; MMEF, maximum mid-expiratory flow; PEF, peak expiratory flow.

to the diagnostic criteria, they were divided into asthma $(n=142)$, COPD $(n=160)$ or ACO $(n=72)$ groups. The mean age and smoker ratio in the three groups were significantly different from each other $(p<0.001$ and $p<0.007$, respectively). The male ratio was significantly decreased in the asthma group when compared with the levels in the COPD and ACO groups $(p<0.001$ and $p<0.001$, respectively); however, the male ratio in the COPD and ACO groups were not statistically significant $(p=0.119)$. All participants were Chinese.

PFT results are reported in Table 2. PFT values had a highly statistically significant difference among different groups ( $p<0.001$ ), except FVC between the asthma and ACO groups $(p=0.378)$.

\section{Sputum induction}

Eosinophilic airway inflammation was defined as sputum eosinophilia $\geq 2.5 \%$. The results of induced sputum cells are provided in Table 3. The sputum levels of eosinophilic percentage were significantly decreased in the COPD group when compared with those in the asthma and ACO groups ( $p<0.001$ and $p<0.001$, respectively). In addition, sputum neutrophil $\%$ and macrophage $\%$ count had a highly statistically significant difference between the groups $(p<0.001$ and $p<0.001$, respectively). There was no significant difference in sputum eosinophil\% and lymphocyte \% count between the ACO and asthma groups ( $p=0.668$ and $p=0.327$, respectively).

The results of induced sputum cells in the stage of COPD, ACO and asthma are provided in Table 4. There was no significant difference in the percentage of induced sputum cells between the stage of airway obstruction and the stage of BHR.

\section{Correlation between sputum eosinophils in $\mathrm{BHR}$ and bronchial reversibility}

A significant correlation was found between sputum eosinophils and $\mathrm{PD}_{20}(\mathrm{mg})$ in the asthma group $(\mathrm{r}=-0.1683$; $p=0.0453$; Figure 1A). However, no significant association was observed between $\Delta \mathrm{FEV}_{1}(\mathrm{~mL})$ and sputum eosinophils $(\mathrm{r}=0.1498 ; p=0.2124$; Figure 1B). There was no relationship between sputum eosinophil\% count in BHR and bronchodilator reversibility ( $p=0.921$ and $p=0.085$, respectively).

\section{Analysis of the correlation between induced sputum cell counts as the dependent factor and clinical variables in the asthma, COPD and ACO groups}

The results of ROC analysis for induced sputum cells are provided in Table 5. Sputum eosinophil\% count could

Table 3 Results of induced sputum cells of the patients

\begin{tabular}{lllll}
\hline Variables & Asthma (N=I42) & COPD (N=I60) & ACO (N=72) & P-value \\
\hline Eosinophil (\%) & $3.93(1.05,21.18)$ & $1.5(0.38,4.01)$ & $5.12(1.37,18.86)$ & $<0.00 I^{*}$ \\
Neutrophil (\%) & $71.09(42.9,90.74)$ & $92.18(85.35,96.08)$ & $80.5(61.2,91.1)$ & $<0.001$ \\
Lymphocyte (\%) & $0.87(0.2,1.62)$ & $1.3(0.58,2.7)$ & $1.1(0.4,1.82)$ & $0.005^{*}$ \\
Macrophage (\%) & $9.8(3.16,30.15)$ & $2.3(0.95,5.95)$ & $5.74(2.07,16.08)$ & $<0.001$ \\
\hline
\end{tabular}

Notes: Values are expressed as median (interquartile range). *Difference between two groups was not statistically significant ( $p>0.05$ ), $p$-value for the differences was analyzed by Kruskal-Wallis test.

Abbreviation: ACO, asthma-COPD overlap. 


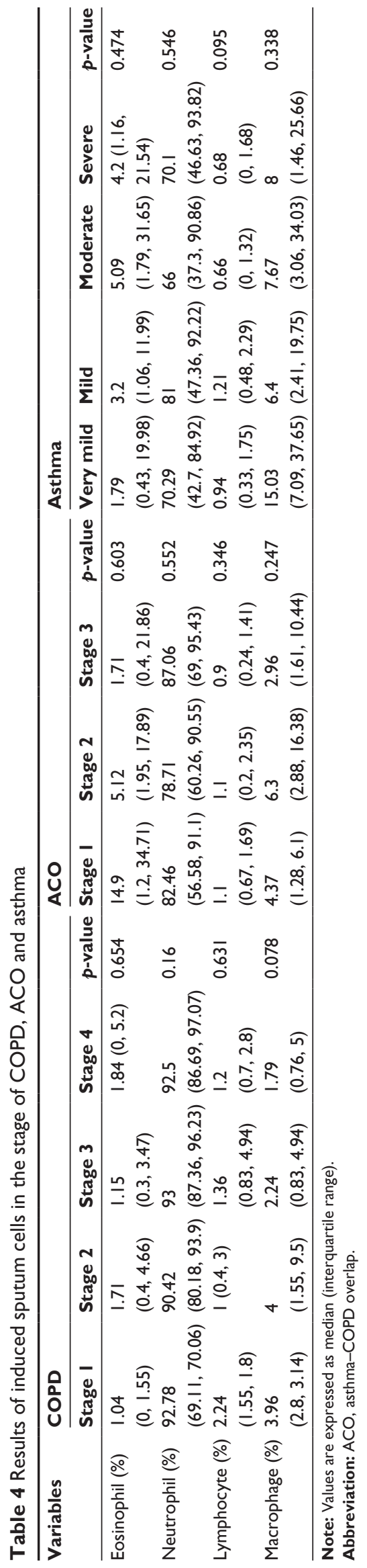

differentiate patients with COPD from patients with asthma and ACO with an area under the curve (AUC) value of 0.708 (95\% CI $0.633-0.782, p<0.001)$ and $0.673(95 \%$ CI $0.612-0.733, p<0.001)$, and also neutrophil $\%$ count with an AUC value of $0.729(95 \%$ CI $0.658-0.8, p<0.001)$ and 0.766 (95\% CI 0.712-0.82, $p<0.001$ ), respectively (Figure 2A and $\mathrm{B})$. In addition, sputum macrophage $\%$ count could differentiate asthma group from the ACO group with the best cutoff value of $7.16 \%$ (AUC $0.611 ; 95 \%$ CI $0.534-0.688$, $p<0.008$; Figure 2C).

\section{Discussion}

This study analyzed that the measurement of induced sputum cell counts may provide additional diagnostic information to conventional lung function tests for diagnosing asthma, COPD and ACO, although the sensitivity and specificity of these biomarkers are different. The level of sputum neutrophil\% count was higher in patients with COPD than in patients with asthma and ACO, and the level of sputum eosinophil\% count was lower in patients with COPD than in patients with asthma and ACO, but this was not apparent in patients with asthma and ACO. In both cohorts, the sputum level of macrophage $\%$ count was elevated in asthma when compared with those of ACO.

Following the 2014 recommendations of the Chinese National Guidelines on Diagnosis and Management of Cough, eosinophilic airway inflammation was defined as the percentage of eosinophil count $\geq 2.5 \%$ in induced sputum. ${ }^{4}$ As listed in Table 3, airway eosinophilia is not exclusive to asthma and is present in patients with COPD. In addition, the majority of patients with COPD do not exhibit high eosinophil, but a high eosinophil in a COPD patient may indicate that this patient has ACO. Furthermore, the recent treatment guidelines of GINA (2017) presents features that characterize $\mathrm{ACO}$ rather than provide a formal definition of ACO. ${ }^{2}$ Four distinct pathways have been proposed for ACO. Airflow limitation in early life can persist through adolescence into adulthood, and ACO is more likely than severe asthma if other risk factors, such as smoking, are present. A second pathway is represented in COPD patients with substantial smoking histories or other exposures and late-onset features of asthma. A third asthma-dominant pathway is represented by asymptomatic adults with airway hyperresponsiveness who progress to chronic airflow limitation compatible with a diagnosis of COPD. Finally, a fourth pathway recognizes the link between early life risk factors and small lungs, with an increased risk of development of fixed airflow limitation and asthma. Thus, from the molecular genetic point of view, 

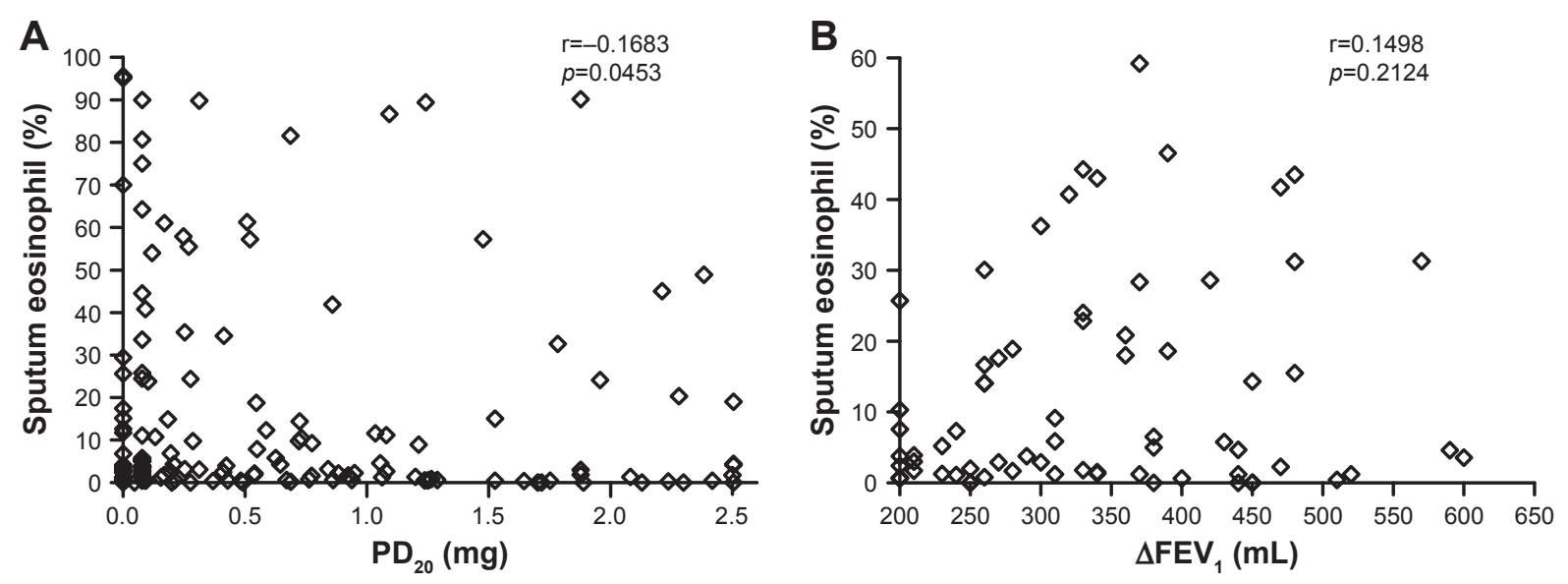

Figure I Scatter plots of correlations between sputum eosinophils, BHR and bronchodilator reversibility.

Notes: (A) Correlation between the percentage of sputum eosinophils and $\mathrm{PD}_{20}(\mathrm{mg})$. (B) Correlation between the percentage of sputum eosinophils and $\Delta \mathrm{FEV}$, (mL); $\Delta$, improvement in FEV, after $400 \mu \mathrm{g}$ of salbutamol.

Abbreviations: $\mathrm{BHR}$, bronchial hyperresponsiveness; $\mathrm{FEV}_{1}$, forced expiratory volume in I second; $\mathrm{PD}_{20}$, provocative dose.

ACO can represent a very heterogeneous group of patients. In addition, from clinical and practical point of view, ACO is characterized by persistent airflow limitation and airway reversibility with several features usually associated with both asthma and COPD. ${ }^{6}$

The GOLD (2017) recently published an evidence-based clinical research guideline that blood eosinophilic counts/ percentage may be a biomarker of exacerbation risk in patients with a history of exacerbation and can predict the effects of ICS on exacerbation prevention. ${ }^{3}$ One study of stable COPD has shown that peripheral blood eosinophil count $\left(\geq 0.3 \times 10^{9} / \mathrm{L}\right)$ can serve as a diagnostic biomarker of sputum eosinophilia ( $\geq 3 \%$; AUC 0.76 [95\% CI 0.67-0.84]; sensitivity $76 \%$; specificity $60 \%)^{7}$

The important finding of the study indicated that sputum cell counts provide additional diagnostic information for asthma, COPD and ACO. Therefore, although the three diseases might be complex and heterogeneous, the use of sputum may improve their specificity for airway inflammation. The changes in sputum cell count levels are likely to reflect the changes of cell composition and lung function during the development of chronic airway disease. Therefore, this study results suggest that ACO could be characterized by mediators related to airway inflammation and airway remodeling.

The strength of this study design was that the diagnostic criteria for asthma, $\mathrm{COPD}$, and $\mathrm{ACO}$ were based on international guidelines, sputum cell counts and clinical characteristics of these diseases. In addition, we excluded patients who had characteristics possibly affecting biomarker measurement, including oral/ICS users and a confounding pulmonary comorbidity.

Table 5 Results of ROC analysis of induced sputum cells in the discovery cohort

\begin{tabular}{|c|c|c|c|c|}
\hline Discovery cohort & Eosinophil (\%) & Neutrophil (\%) & Lymphocyte (\%) & Macrophage (\%) \\
\hline \multicolumn{5}{|l|}{ ACO versus COPD } \\
\hline $\operatorname{AUC}(95 \% \mathrm{Cl})$ & $0.708(0.633-0.782)$ & 0.27 I (0.2-0.342) & $0.433(0.354-0.513)$ & $0.660(0.583-0.736)$ \\
\hline Cutoff (\%) & 3.55 & 84.55 & 5.76 & 4.63 \\
\hline Sensitivity/specificity (\%) & $59.7 / 72.5$ & $37.5 / 23.7$ & $97 / 12$ & $58.3 / 69.4$ \\
\hline$p$-value & $<0.001$ & $<0.001$ & 0.104 & $<0.001$ \\
\hline \multicolumn{5}{|l|}{ Asthma versus COPD } \\
\hline AUC $(95 \% \mathrm{Cl})$ & $0.673(0.6 \mid 2-0.733)$ & $0.234(0.18-0.288)$ & $0.393(0.329-0.456)$ & $0.746(0.69-0.802)$ \\
\hline Cutoff $(\%)$ & 8.95 & 76.4 & 1.77 & 5.33 \\
\hline Sensitivity/specificity (\%) & $38.7 / 88.7$ & $44.4 / 11.2$ & $22.5 / 60$ & $65.5 / 72.5$ \\
\hline$p$-value & $<0.001$ & $<0.001$ & 0.001 & $<0.001$ \\
\hline \multicolumn{5}{|l|}{$A C O$ versus asthma } \\
\hline AUC $(95 \% \mathrm{Cl})$ & $0.518(0.438-0.598)$ & $0.585(0.508-0.66 \mathrm{I})$ & $0.54 I(0.458-0.623)$ & $0.389(0.312-0.466)$ \\
\hline Cutoff $(\%)$ & 1.11 & 70.66 & 1.64 & 7.16 \\
\hline Sensitivity/specificity (\%) & $84.7 / 25.4$ & $69.4 / 50$ & $34.7 / 76.1$ & $36.1 / 43$ \\
\hline$p$-value & 0.668 & 0.43 & 0.33 & 0.008 \\
\hline
\end{tabular}

Abbreviations: ACO, asthma-COPD overlap; AUC, area under the curve; ROC, receiver operating characteristic. 
A

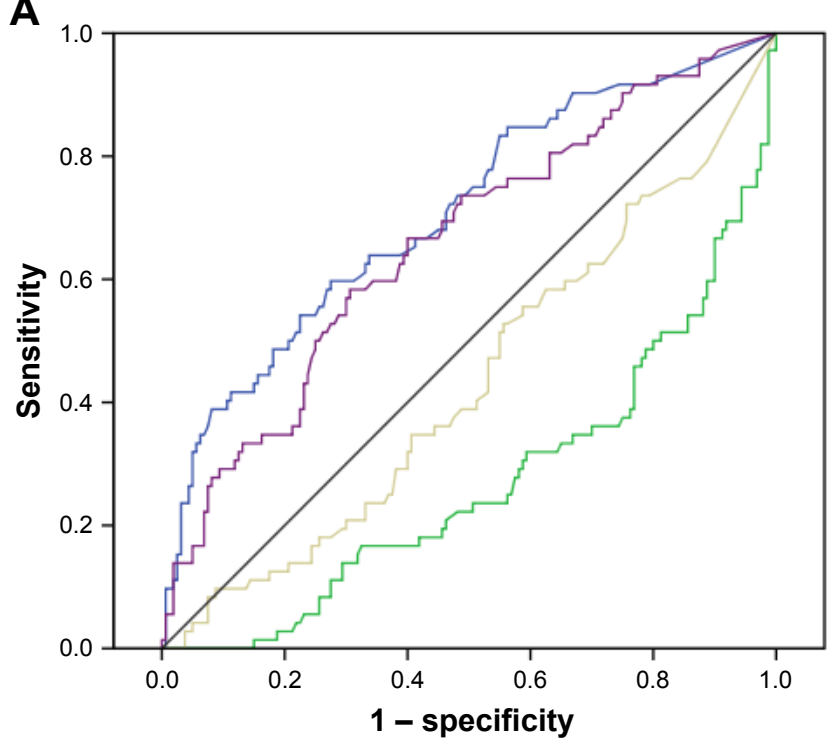

B

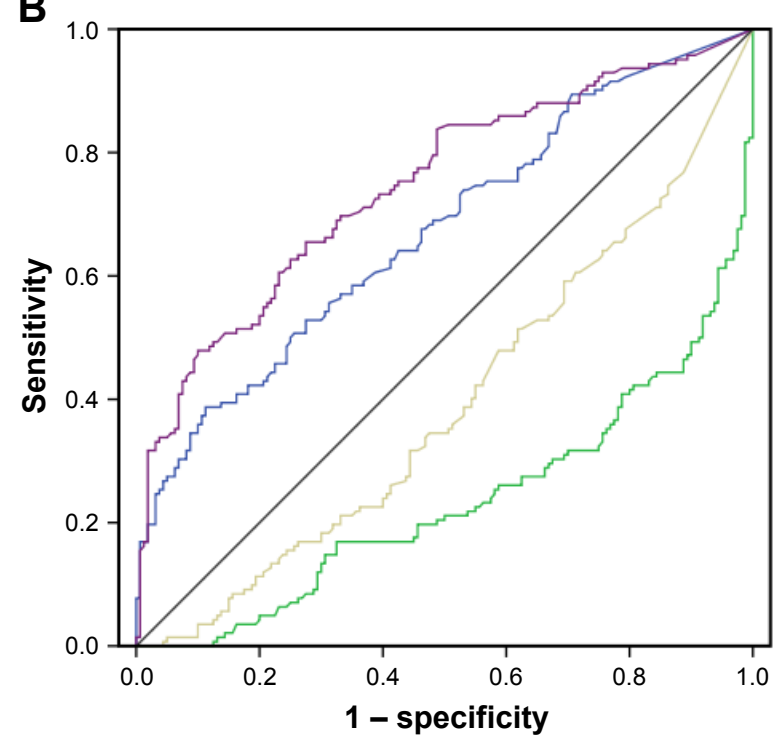

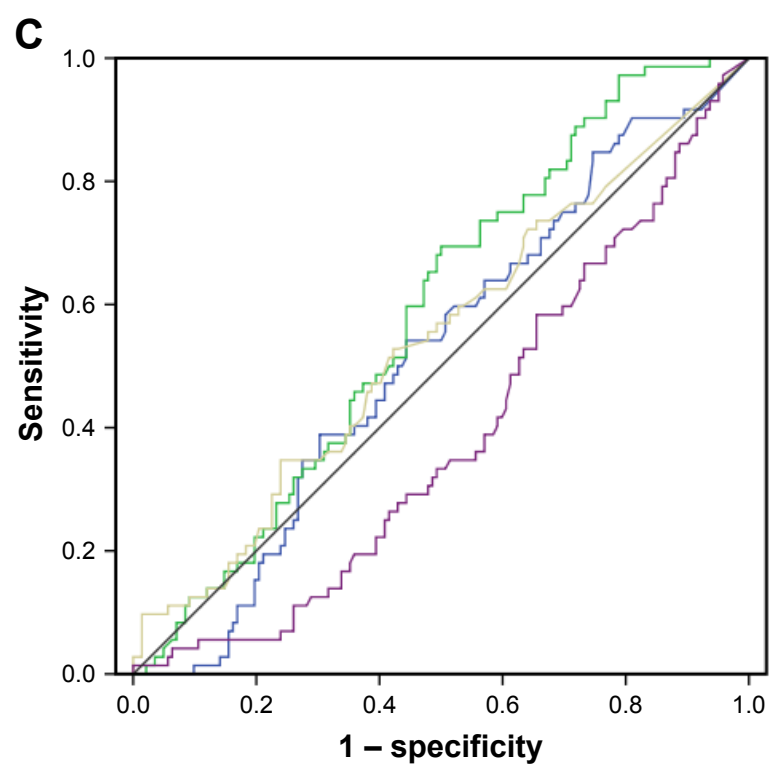

- Eosinophil (\%) - Neutrophil (\%) - Lymphocyte (\%) - Macrophage (\%) — Reference line

Figure 2 ROC curve for induced sputum cell counts in ACO versus COPD (A), asthma versus COPD (B), ACO versus asthma (C). Abbreviations: ACO, asthma-COPD overlap; ROC, receiver operating characteristic.

\section{Limitations}

A limitation of the study was that the $p$-value $(p=0.0453)$ changed slightly under 0.05 , and the correlation coefficients $(\mathrm{r}=-0.1683)$ were very low, as shown in Figure 1A. The given number of data points may not be taken as strong evidence for such a relationship. It is possible that no relevant correlation between sputum eosinophil $\%$ and $\mathrm{PD}_{20}$ exists in the clinical practice.

\section{Conclusion}

The clinical relevance of this study provides evidence that $\mathrm{ACO}$ is associated with sputum biomarkers that drive both airway inflammation and airway inflammation phenotypes, and these biomarkers could carry some information to distinguish ACO, asthma and COPD. These data may be useful for identifying patients with airway inflammation phenotypes who will have a beneficial response to treatment with an ICS, and it is important to help guide treatment and management of COPD patients. However, we need additional large-scale prospective studies to expand the clinical application of the measurement of induced sputum cell counts.

\section{Disclosure}

The authors report no conflicts of interest in this work. 


\section{References}

1. Eltboli O, Brightling CE. Eosinophils as diagnostic tools in chronic lung disease. Expert Rev Respir Med. 2013;7(1):33-42.

2. Global Initiative for Asthma [webpage on the Internet]. Global Strategy for Asthma Management and Prevention. Available from: http://ginasthma.org/2017-pocket-guide-for-asthma-management-andprevention/. Accessed January 19, 2017.

3. Global Initiative for Chronic Obstructive Lung Disease (GOLD) [webpage on the Internet]. The Global Strategy for the Diagnosis, Management and Prevention of COPD. 2017. Available from: http://www.goldcopd. org/. Accessed October 16, 2016.
4. The Chinese national guidelines on diagnosis and management of cough. Chin Med J. 2016;39(5):321-339.

5. The Chinese national guidelines of pulmonary function test. Chin $J$ Tuberc Respir Dis. 2014;37(8):566-571.

6. Gao J, Iwamoto H, Koskela J, et al. Characterization of sputum biomarkers for asthma-COPD overlap syndrome. Int J Chron Obstruct Pulmon Dis. 2016;30(11):2457-2465.

7. Negewo NA, McDonald VM, Baines KJ, et al. Peripheral blood eosinophils: a surrogate marker for airway eosinophilia in stable COPD. Int J Chron Obstruct Pulmon Dis. 2016;1(11):1495-1504.

\section{Publish your work in this journal}

The International Journal of COPD is an international, peer-reviewed journal of therapeutics and pharmacology focusing on concise rapid reporting of clinical studies and reviews in COPD. Special focus is given to the pathophysiological processes underlying the disease, intervention programs, patient focused education, and self management protocols.

\section{Dovepress}

This journal is indexed on PubMed Central, MedLine and CAS. The manuscript management system is completely online and includes a very quick and fair peer-review system, which is all easy to use. Visit $\mathrm{http} / / / \mathrm{www}$.dovepress.com/testimonials.php to read real quotes from published authors. 